\title{
THE BIOMECHANICS OF FAST-STARTS DURING ONTOGENY IN THE COMMON CARP CYPRINUS CARPIO
}

\author{
JAMES M. WAKELING*, KIRSTY M. KEMP AND IAN A. JOHNSTON \\ Gatty Marine Laboratory, School of Environmental and Evolutionary Biology, University of St Andrews, St Andrews, \\ Fife KY16 8LB, UK \\ *Present address: Human Performance Laboratory, The University of Calgary, Calgary, Alberta, Canada T2N 1N4 \\ (e-mail: jmw5@st-andrews.ac.uk)
}

Accepted 1 September; published on WWW 28 October 1999

\begin{abstract}
Summary
Common carp Cyprinus carpio L. were reared a constant temperature of $20^{\circ} \mathrm{C}$ from the larval ( $7 \mathrm{~mm}$ total length) to the juvenile $(80 \mathrm{~mm})$ stage. Body morphology and white muscle mass distribution were measured. Fast-start escape responses were recorded using high-speed cinematography from which the velocities, accelerations and hydrodynamic power requirements were estimated. All three measures of fast-start performance increased during development. White muscle contraction regimes were calculated from changes in body shape during the fast-starts and used to predict the muscle force and power production for all longitudinal positions along the body. Scaling arguments predicted that increases in body length would constrain the fish to bend less rapidly because the cross-sectional muscle area, and hence force production, does not increase at the same rate as the inertial mass that resists bending. As

mechanical power produced by the muscles to the inertial power requirements in the direction of travel, showed no significant change during ontogeny. The increasing hydrodynamic power requirements were thus met by increases in the power available from the muscles. The majority of the increases in fast-start swimming performance during ontogeny can be explained by sizedependent increases in muscle power output. For all sizes, there was a decrease in muscle-mass-specific power output and an increase in muscle stress in a posterior direction along the body due to systematic variations in fibre strain. These changing strain regimes result in the central muscle bulk producing the majority of the power requirements during the fast-start, and this power is transmitted to the tail region of the fish and ultimately to the water via muscle in the caudal myotomes.
\end{abstract} predicted, the increases in body length resulted in decreases in muscle shortening velocity, and this coincided with increases in both the force and power produced by the muscles. The hydrodynamic efficiency, which relates the

Key words: fast-start, swimming, hydrodynamics, muscle, power, force, development, scaling, carp, Cyprinus carpio.

\section{Introduction}

As fish develop from larvae into adults, they undergo a series of changes in body morphology, muscle dynamics and swimming kinematics (Osse, 1990). Larvae are typically small, and their body depth is reasonably uniform because of the primordial fin. Adult fish, in contrast, are large and have an irregular depth because of changes in both body profile and the size and shape of the median fins. The changes in size and shape during ontogeny result in changes in swimming hydrodynamics and kinematics which, in turn, impose differing demands on the muscle contractile dynamics.

The short body length and low swimming velocities of larvae can result in their swimming being dominated by the viscous forces of low-Reynolds-number regimes. The Reynolds number $R e$ is given by:

$$
R e=V L / v,
$$

where $V$ is the swimming velocity, $L$ is the total fish length and

$v$ is the kinematic viscosity of the water. Larvae swimming at $100<R e<300$ experience significant viscous effects of the water (Fuiman and Batty, 1997). They typically swim with an 'anguilliform' undulatory mode of propulsion in which the whole body participates (Lighthill, 1969, 1970). The body depth along a larva is relatively uniform because of a primordial fin-fold running along both the dorsal and ventral surfaces of the body; however, this fin-fold may have little locomotor function during early development (Webb and Weihs, 1986). As the fish develop, they swim in regimes of higher Reynolds number, and ultimately, as adults, they routinely swim in the inertial hydrodynamic regime with $R e>10^{4}$ (Wu, 1977). In this regime, efficient swimming can be achieved when the lateral body oscillations are confined to the rear region of the fish in a 'carangiform' style of swimming, and increases in efficiency are achieved by modifications to the body depth by caudal and median fins stiffened with fin ray 
muscles and spines (Lighthill, 1969, 1970; Osse, 1990). However, fast-start performance in adult fish is the result of a compromise between the longitudinal depth distribution and the distribution of muscle mass (Webb, 1978).

Swimming velocity increases with body size both for steady prolonged activity (for a review, see Videler, 1993) and for burst activity and fast-starts (e.g. Beamish, 1978; Magnuson, 1978; Hale, 1996; Williams and Brown, 1992; Temple and Johnston, 1998; James and Johnston, 1998a). During fast-start swimming, the mass-specific inertial power requirements are related to the product of velocity and acceleration (Frith and Blake, 1995; Wakeling and Johnston, 1998) and thus also increase with size. Hydrodynamic power requirements for fast-starts are limited by the power available from the muscles (Wakeling and Johnston, 1998) which, in turn, must increase for bigger fish. These increases in the mass-specific power output from the muscle may be due to differences in contractile properties or to the differences in the imposed contraction regimes resulting from the changing kinematics of swimming.

Muscle contractile properties scale with body length in fish. The $\mathrm{Q}_{10 \mathrm{~cm}}\left(\mathrm{a} \mathrm{Q}_{10}\right.$ value for every $10 \mathrm{~cm}$ difference in length; Videler and Wardle, 1991) for twitch contraction frequencies was measured at 0.82-0.94 for cod Gadus morhua (Archer et al., 1990; Videler and Wardle, 1991). A $Q_{10 \mathrm{~cm}}$ of 0.79 has been measured for the maximum unloaded shortening velocity of fast muscle fibres in shorthorn sculpin Myoxocephalus scorpius (James and Johnston, 1998b), but no significant scaling relationship was found for this variable in dogfish Scyliorhinus canicula (Curtin and Woledge, 1988). These scaling relationships predict a maximum $11 \%$ change in any individual contractile variable for the initial $5 \mathrm{~cm}$ growth of a fish. It is therefore unlikely that changes in muscle contractile properties contribute in a major way to the increase in fast-start performance during development.

In this study, the body morphologies during the ontogeny of the common carp Cyprinus carpio from a $7 \mathrm{~mm}$ larva to an $80 \mathrm{~mm}$ juvenile were measured. Fast-start escape swimming was captured on high-speed film for a series of size groups within this range, and the swimming kinematics, hydrodynamics and muscle dynamics were quantified for these fast-starts. The size-dependent limitations to the kinematics and muscle dynamics are discussed, and it is shown that the fast-start biomechanics can be explained in terms of the developmental constraints of size and shape.

\section{Materials and methods}

Fish

Three-day-old common carp larvae Cyprinus carpio L., approximately $8 \mathrm{~mm}$ in total length, were supplied by Humberside Fisheries, Driffield, UK, in May 1998. The fish were reared in duplicate temperature-controlled tanks at between 20.4 and $21.1{ }^{\circ} \mathrm{C}$ with a light:dark photoperiod of $12 \mathrm{~h}: 12 \mathrm{~h}$. Fish were fed live Artemia for the first month and were then weened onto proprietary dry food (BOCM Pauls Ltd,
UK). By the end of November 1998, the fish had reached a mean length of $60 \mathrm{~mm}$.

\section{Body morphologies}

Body morphologies were measured using the methods described by Wakeling and Johnston (1998). The following variables were measured. Direct measurements were made for total body length $L$ and body mass $m$. Longitudinal and planform images were used to estimate the distributions of area, volume and mass along the fish, and variables were normalized by length $L$, area $L^{2}$ or volume $L^{3}$. The body-length-specific half-width at any longitudinal position is given by $\hat{b}$, whilst area-specific variables for planform (top-view) area $\hat{S}_{\mathrm{p}}$ and longitudinal (sideview) area $\hat{S}_{1}$ are equivalent to the mean length-specific width and depth of the fish, respectively. Length-specific distances from the snout are denoted $\hat{l}$. The length-specific radius of the first moment of volume from the snout $\hat{l}_{1}(M)$ gives a good estimate of the position of the centre of mass for a straightstretched fish, and the masses for ten equal-length longitudinal segments were also estimated.

Freshly killed fish $(N=35,7.8<L<62.0 \mathrm{~mm})$ were frozen in isopentane cooled to $-159^{\circ} \mathrm{C}$ in liquid nitrogen. Fish were divided into ten segments of equal length, which were set in Cryomatrix embedding medium (Shandon, PA, USA) and mounted onto a cryostat chuck. Transverse sections, $10 \mu \mathrm{m}$ thick, were cut through the centre of each segment, mounted on glass slides and stained with Harris haematoxylin solution (Sigma-Aldrich Co. Ltd, UK). The cross-sectional area of the white muscle in each section was then determined using a microscope drawing arm and Videoplan image-analysis software (Kontron, Eching, Germany). The white muscle mass for each segment was calculated from its cross-sectional area and the segment depth, assuming a muscle density of $1.06 \mathrm{mg} \mathrm{mm}^{-3}$ (Mendez and Keys, 1960).

The body-mass-specific white muscle mass $\hat{m}_{\mathrm{m}}$ was the ratio of the total white muscle mass to $m$.

\section{Filming and kinematic analysis}

Fast-starts of the carp were filmed in a temperaturecontrolled tank measuring $500 \mathrm{~mm} \times 500 \mathrm{~mm} \times 180 \mathrm{~mm}$ (length $\times$ width $\times$ depth). Fast-starts were elicited by thrusting an obliquely angled fine rod towards the snout of the fish. In all cases, the fish responded without making contact with the rod, and images from the fast-start were obtained before the water ripple from the rod passed over the fish. A frosted sheet beneath the tank was illuminated by a $250 \mathrm{~W}$ projector bulb. Overhead high-speed images of the fish were taken via a $45^{\circ}$ mirror using both video (NAC Inc., Japan, HSV-500 C3 VCR with $\mathrm{C} 3$ digital high-speed video camera) and ciné (NAC E-10 camera, Ilford HP5 $16 \mathrm{~mm}$ film) equipment at frame rates of $500 \mathrm{~s}^{-1}$ and $300 \mathrm{~s}^{-1}$, respectively. The light path between the fish and the cameras was $3.4 \mathrm{~m}$, and zoom lenses were set so that the image diagonal corresponded to approximately eight fish lengths in both cases. Video sequences were digitized via a Win/TV image grabber card (Hauppauge, UK), whilst ciné sequences were analyzed on a NAC $160 \mathrm{~F}$ film motion image 
analyzer. The standard error of digitizing a reference point on sequential frames corresponded to 0.004 and $0.002 L$ for the video and ciné films, respectively. A $100 \mathrm{~mm}$ diameter circle was drawn on the underside of the tank and was used to calibrate the aspect ratio for each filming method.

The kinematic analysis of the swimming sequences followed the methods described by Wakeling and Johnston (1998) unless stated otherwise. The following description is only a summary of these methods.

Ten points along the midline of the fish were digitized for each frame, from the head to the tail. These points were positioned equidistant between the two sides of the fish silhouette. The spine position was calculated as a continuous spline passing through these points. The mean spine length $L$ from the entire swimming sequence was used as a reference length for subsequent calculations. The standard error of the mean spine length for all sequences was less than $0.01 L$ and was typically $0.006 L$. The centre of mass for each frame was calculated using the coordinates of the centres of ten segments of equal length along the fish and the masses of those segments (see above). The length-specific velocity $\hat{V}$ and length-specific acceleration $\hat{A}$ were calculated from the displacement of the centre of mass. Position data were smoothed using piecewise cubic regressions, and a mean appropriate smooth width was determined for each size group tested: this smooth width varies with fish image size, filming and digitizing method, the filming frame rate and the actual swimming patterns of the fish. The useful inertial power requirement for each fast-start $P_{\mathrm{i}}$ is the product of the component of force acting in the direction of travel and the swimming velocity. The force requirements for the start are, in turn, the product of mass and acceleration so that:

$$
P_{\mathrm{i}}=\left(m+m_{\mathrm{a}}\right) A V \cos \phi,
$$

where $m$ is fish mass, $m_{\mathrm{a}}$ is the added mass of water that must be accelerated with the fish, $A(=\hat{A} L)$ is acceleration, $V(=\hat{V} L)$ is velocity and $\phi$ is the angle between the velocity and acceleration vectors.

The radius of curvature $r$ was calculated for all positions along the spine. In this study, spine bending is expressed as length-specific curvature $\hat{c}(=r / L)$ and takes the convention of positive values when bending is to the right and negative values when bending is to the left.

Contour plots of spine curvature against both body position and time showed that the initial body bend during the fast-start propagated as a wave of curvature travelling in a posterior direction along the spine. The length-specific velocity $\hat{U}$ of the wave of curvature along the spine was given by the slope of the best-fitting straight line that connected the initial crest on such contour plots.

The mean white muscle strain $\varepsilon_{\mathrm{w}}$ for any section across the fish was calculated from the product of the spine curvature $\hat{c}$ through that section, the maximum length-specific half-width of that section $\hat{b}$, and the gearing ratio $\lambda$ where:

$$
\varepsilon_{\mathrm{W}}=\hat{b} \hat{c} / \lambda \text {. }
$$

The gearing ratio takes values ranging from 1.94 to 2.63 , and its value varies as a function of longitudinal position along the trunk (Wakeling and Johnston, 1999a).

\section{Modelling in vivo muscle dynamics}

The force development of the white muscle fibres during the initial tail-beat of the fast-starts was determined using the methods described by Wakeling and Johnston (1999b). For each fast-start sequence, the contraction duration and change in muscle strain were determined for seven longitudinal positions between $0.3 \mathrm{~L}$ and $0.8 \mathrm{~L}$, i.e. the region where the white myotomal muscle is situated. The initial muscle contraction of a fast-start is initiated by electromyographic (EMG) activity at the onset of shortening (Wakeling and Johnston, 1999c). The muscle length changes were assumed to be sinusoidal in shape, with strain amplitude and contraction durations matching those measured for the swimming fish. The predictive model for muscle force (Wakeling and Johnston, 1999b) generates force and power estimations that are statistically indistinguishable from measured force and power from live fibre preparations during realistic fast-start contraction regimes. The contractile variables used in the model correspond to those for white muscle fibres in adult carp $(L=130 \mathrm{~mm})$ at $20^{\circ} \mathrm{C}$, i.e. maximum isometric stress at $229.7 \mathrm{kN} \mathrm{m}^{-2}$ and maximum length-specific unloaded contraction velocity at $19.65 \mathrm{~s}^{-1}$ (Wakeling and Johnston, 1999b). The stimulus duration was numerically optimized for each contraction to yield the mean maximum power output, and the optimal durations ranged between 2.0 and $9.3 \mathrm{~ms}$.

The total muscle power output $\overline{P_{\mathrm{m}, \mathrm{t}}}$ during each fast-start was calculated as the mean power output generated at all sites along the myotome weighted by the proportion of white muscle mass located at each of those sites.

\section{Statistics}

Mean values are presented with the standard error of the mean (S.E.M.). Scaling relationships were calculated from the least-squares regression lines passing through log-transformed data. The effects of body length and longitudinal position along the trunk on the force and power output from the myotomal muscle were tested with an analysis of covariance (ANCOVA) where length and position were factors in the model. Significance testing was accepted at a $95 \%$ confidence level.

\section{Results \\ Body morphologies}

Body morphologies were determined for 104 fish ranging in length from 7 to $80 \mathrm{~mm}$. The growth rate of these fish is shown in Fig. 1, and the relationship between body mass and total body length is shown in Fig. 2 . The body mass $m(\mathrm{~kg})$ can be predicted from the total length $L(\mathrm{~m})$ using the following equation calculated from the linear regression through logtransformed data:

$$
m=32.17 L^{3.24},
$$

$\left(N=104, \quad r^{2}>0.99, \quad P<0.0001\right)$. The scaling exponent is 


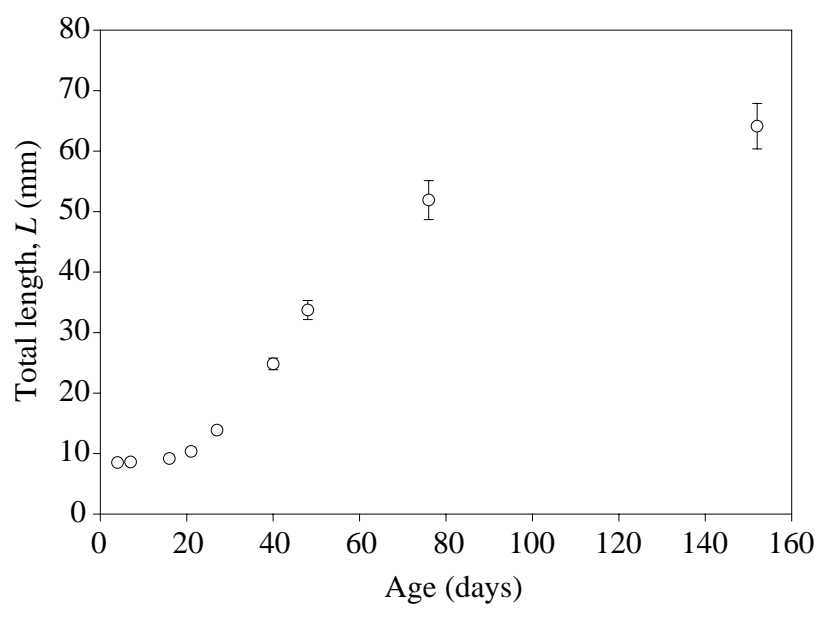

Fig. 1. The total length $L$ of the carp as a function of their age. Values are means \pm S.E.M. $(N=6-17)$.

greater than 3 because body growth across this range is allometric.

The $10 \mathrm{~mm}$ fish were surrounded by primordial fin-folds and showed a reasonably regular body depth along their length. Growth up to $30 \mathrm{~mm}$ proceeded with a gradual reduction of these fin-folds, but with development of the median and caudal fins. In this range, the body increased in both relative width and depth across the mid-region of the fish. Growth beyond $30 \mathrm{~mm}$ was geometric, with the fish retaining their relative body proportions, which were adult in form. These changes are shown in Fig. 3. Developmental changes in non-dimensional longitudinal area $\hat{S}_{1}$ and planform area $\hat{S}_{\mathrm{p}}$ are shown in Fig. 4. The increases in $\hat{S}_{1}$ between $8 \mathrm{~mm}$ and $30 \mathrm{~mm}$ length are due to increases in both body depth and median fin area. Developmental changes in the length-specific distance of the centre of mass from the snout $\hat{l}_{1}(M)$ are shown in Fig. 5 and again show a gradual increase between 8 and $30 \mathrm{~mm}$ length, reaching a plateau in larger fish. This increase in $\hat{l}_{1}(M)$ is due to the increase in both relative width and depth across the midregion of the fish.

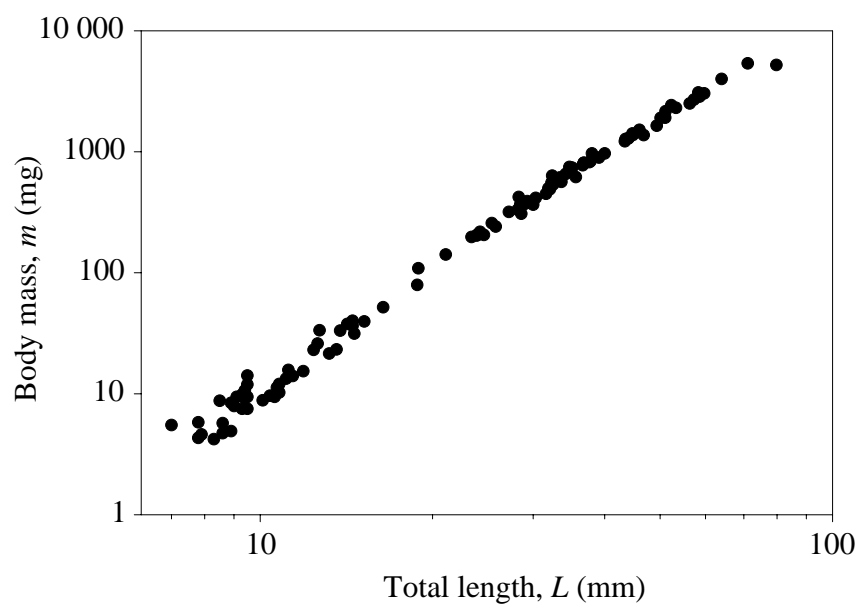

Fig. 2. The body mass of the carp as a function of their total length $L$.
Longitudinal outline Planform outline length, $L$

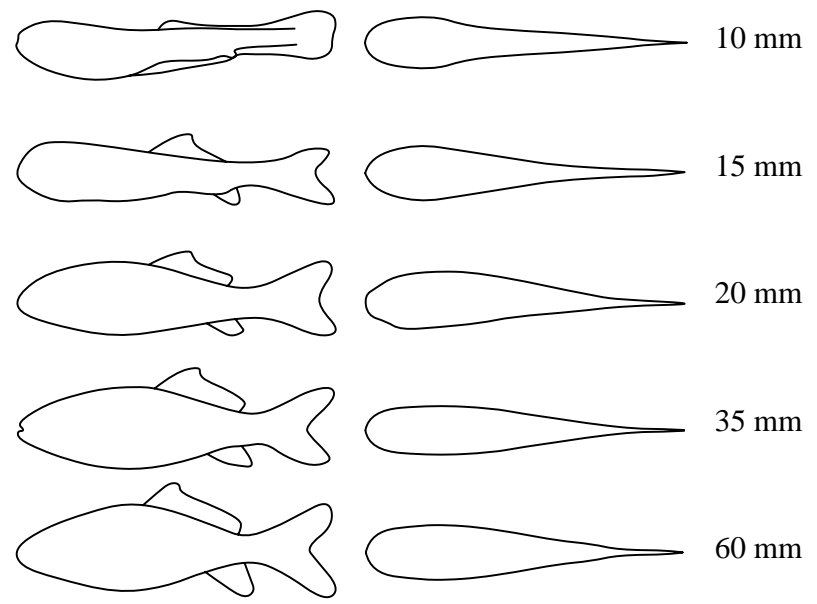

Fig. 3. Longitudinal and planform outlines of the fish at various stages in their development. The drawings have been scaled to the same length.

The longitudinal distribution of white muscle mass is shown in Fig. 6. The $10 \mathrm{~mm}$ fish had the most uniform distribution of mass along their length $(0.2 L-0.8 L)$, and increases in fish length occurred with increases in the proportion of white muscle in the central body region $(0.3 L-0.6 L)$. The $10 \mathrm{~mm}$ fish had the lowest relative muscle mass $\hat{m}_{\mathrm{m}}$ of $0.197 \pm 0.027$ $(N=10) . \hat{m}_{\mathrm{m}}$ increased over the range $10<L<40 \mathrm{~mm}$. For lengths greater than $40 \mathrm{~mm}$, there was no significant effect of length on $\hat{m}_{\mathrm{m}}\left(\hat{m}_{\mathrm{m}}=0.296 \pm 0.022, N=18\right)$.

\section{Fast-start kinematics}

In total, 102 fast-starts were analyzed. These were sorted by fish length into seven groups. From each group, the five starts with the greatest mean hydrodynamic power requirements were selected and represent the best third of the total analyzed

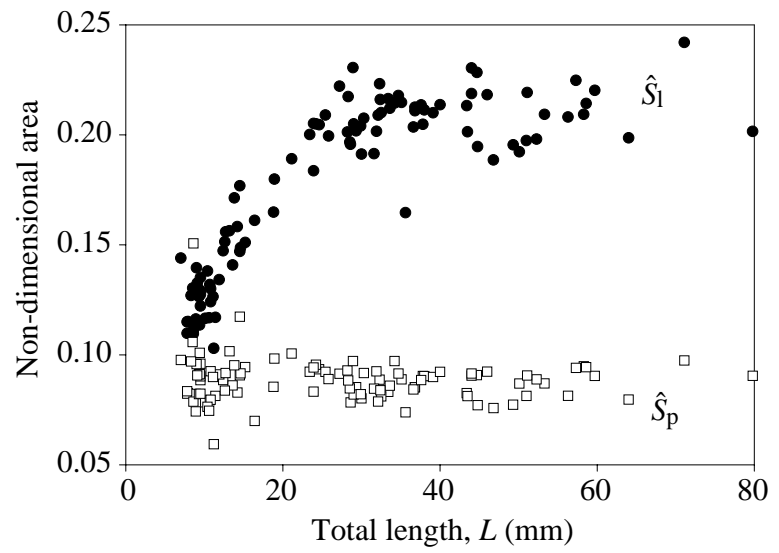

Fig. 4. Changes in non-dimensional longitudinal area $\hat{S}_{1}$ with fins extended (filled circles) and planform area $\hat{S}_{\mathrm{p}}$ (open squares) with increasing body length $L$. The adult shape is acquired by fish of length $30 \mathrm{~mm}$. 


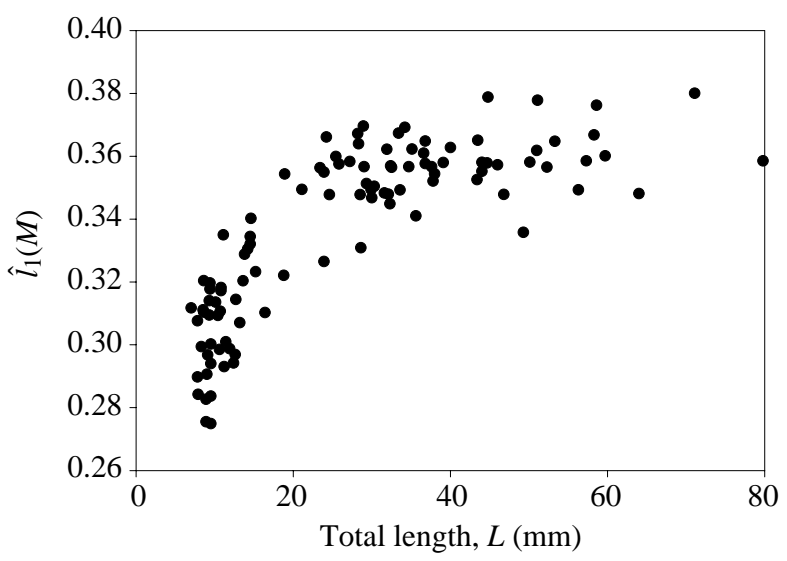

Fig. 5. Changes in length-specific distance of the centre of mass from the snout for straight-stretched fish $\hat{l}_{1}(M)$ with increasing body length. The adult shape is acquired by fish of length $30 \mathrm{~mm}$.

sequences. The mean ( \pm S.E.M.) lengths for these groups were: $9.9 \pm 0.22, \quad 11.6 \pm 0.59, \quad 21.6 \pm 0.64, \quad 25.2 \pm 0.54, \quad 38.7 \pm 1.03$, $50.7 \pm 1.76$ and $62.5 \pm 3.29 \mathrm{~mm}$.

The maximum velocity $V_{\max }$ and acceleration $A_{\max }$ achieved during the first tail-beat both showed an increase with body length (Fig. 7A,B). The body-mass-specific mean power required to accelerate the centre of mass in the direction of travel also showed an increase with fish length from $1.26 \pm 0.16 \mathrm{~W} \mathrm{~kg}^{-1}$ for $L=10 \mathrm{~mm}$ to $6.97 \pm 1.13 \mathrm{~W} \mathrm{~kg}^{-1}$ for $L=63 \mathrm{~mm}$ (Fig. 8). Scaling relationships for these variables were calculated from log-transformed data for each individual start and are given in Table 1.

The length-specific maximum velocity $\hat{V}_{\max }$ and maximum

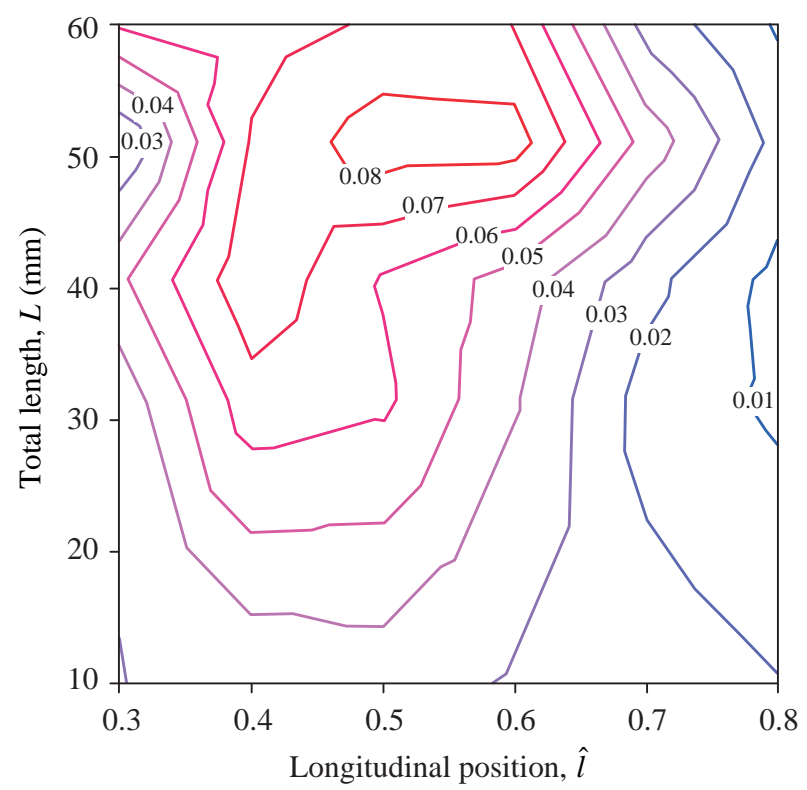

Fig. 6. White muscle mass distribution along the fish. The bodymass-specific muscle mass within each $0.1 \mathrm{~L}$ segment is given by the contour height $(0.01-0.08)$ corresponding to that length-specific longitudinal position. $L$, total length.
Table 1. Scaling exponents for the kinematic variables with variation in body length $(\mathrm{m})$

\begin{tabular}{lcccccc}
\hline Variable & Symbol & $a$ & $b$ & $r^{2}$ & $P$ \\
\hline $\begin{array}{l}\text { Maximum velocity } \\
\left(\mathrm{m} \mathrm{s}^{-1}\right)\end{array}$ & $V_{\max }$ & 3.71 & 0.548 & 0.79 & $<0.001$ \\
$\begin{array}{l}\text { Maximum acceleration } \\
\left(\mathrm{m} \mathrm{s}^{-2}\right)\end{array}$ & $A_{\max }$ & 55.34 & 0.340 & 0.40 & 0.002 \\
$\begin{array}{l}\text { Body-mass-specific power } \\
\text { requirements }\left(\mathrm{W} \mathrm{kg}^{-1}\right)\end{array}$ & $\overline{P_{\mathrm{i}}}$ & 57.54 & 0.802 & 0.64 & $<0.001$ \\
$\begin{array}{l}\text { Muscle contraction duration } \\
(\mathrm{s})\end{array}$ & $\tau$ & 0.0997 & 0.392 & 0.65 & 0.014 \\
$\quad$ Kinematic variables are of the form $a L^{b}$, where $L$ is body length in \\
\begin{tabular}{l}
$m$. \\
\hline
\end{tabular}
\end{tabular}

acceleration $\hat{A}_{\max }$ achieved during the first tail-beat of the faststart both showed a decrease with increasing fish length (Fig. 9A,B). The velocity of the wave of curvature $\hat{U}$ that travelled in a posterior direction along the spine during the initial body bending always exceeded the maximum forward velocity of the fish and is also shown in Fig. 9A.

The duration of the initial muscle contraction increased with
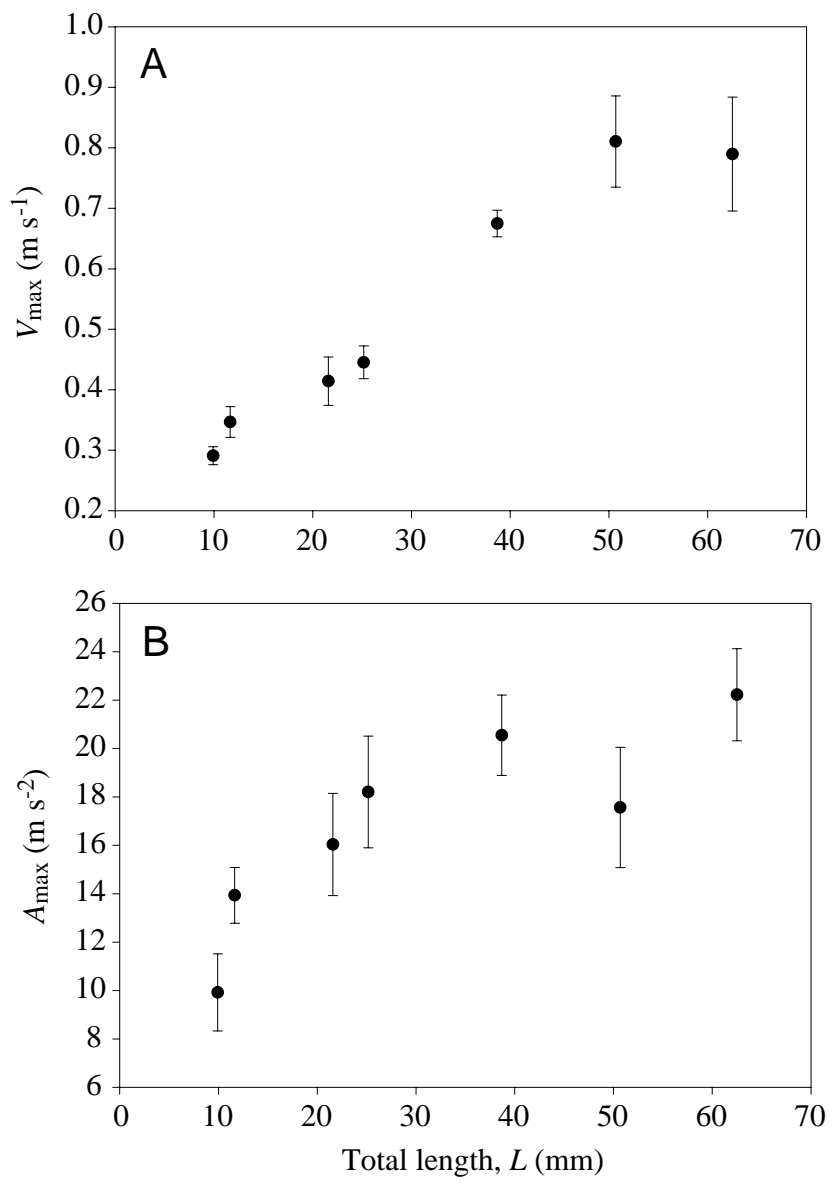

Fig. 7. Maximum velocity $V_{\max }$ (A) and maximum acceleration $A_{\max }$ (B) measured during the first tail-beat of the fast-starts. Symbols denote mean ( \pm S.E.M.) values calculated for the five most powerful fast-starts in each size group. 


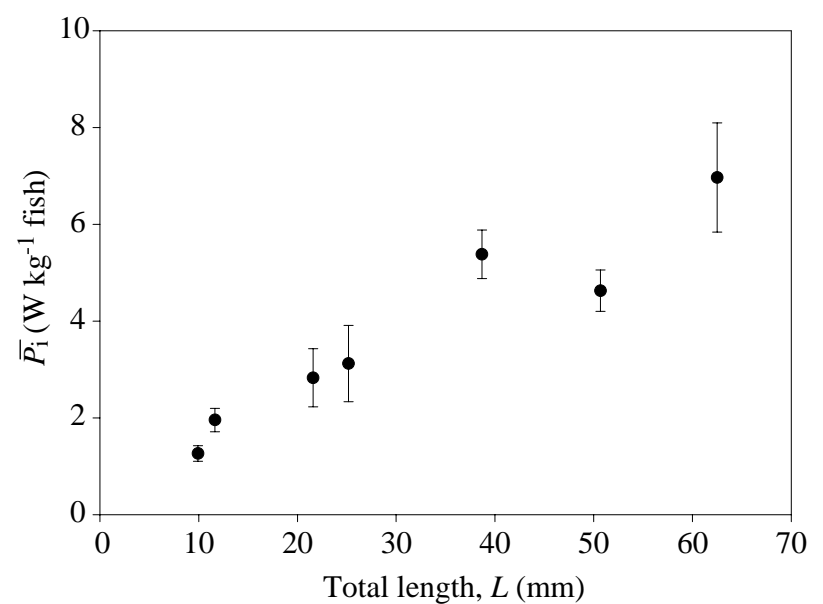

Fig. 8. Mean ( \pm S.E.M.) body-mass-specific hydrodynamic power requirements $\bar{P}_{\mathrm{i}}$ for the fast-starts integrated over the first tail-beat. Values were calculated from the five most powerful fast-starts in each size group.

longitudinal position along each fish, and the time differences for the first peak in muscle strain can be determined from the velocity of the wave of curvature $\hat{U}$ (Fig. 9A). The duration $\tau$ of the next change in peak-to-peak spine curvature and muscle strains remained constant along the length of each fish, and the mean durations of the initial contractions are shown in Fig. 10. $\tau$ increased from $16.9 \pm 0.87 \mathrm{~ms}$ for $L=10 \mathrm{~mm}$ to $34.9 \pm 0.26 \mathrm{~ms}$ for $L=63 \mathrm{~mm}$. The scaling relationship for contraction duration was calculated from log-transformed data for each individual start and is presented in Table 1.

The longitudinal variation of spine curvatures is shown in Fig. 11. The mean curvatures for the $10 \mathrm{~mm}$ fish were lower than for all the larger sizes. In general, the length-specific spine curvature $\hat{c}$ increased with longitudinal position to a value greater than 5 . Spine curvatures were relatively independent of body length for $L \geqslant 12 \mathrm{~mm}$. The longitudinal variation in white muscle strain is shown in Fig. 12. Again, the $10 \mathrm{~mm}$ fish swam using lower strains than the larger fish. For fish of length $L \geqslant 12 \mathrm{~mm}$, the white muscle strain was independent of body length. The general pattern was for the greatest strains $\varepsilon \approx 0.09$
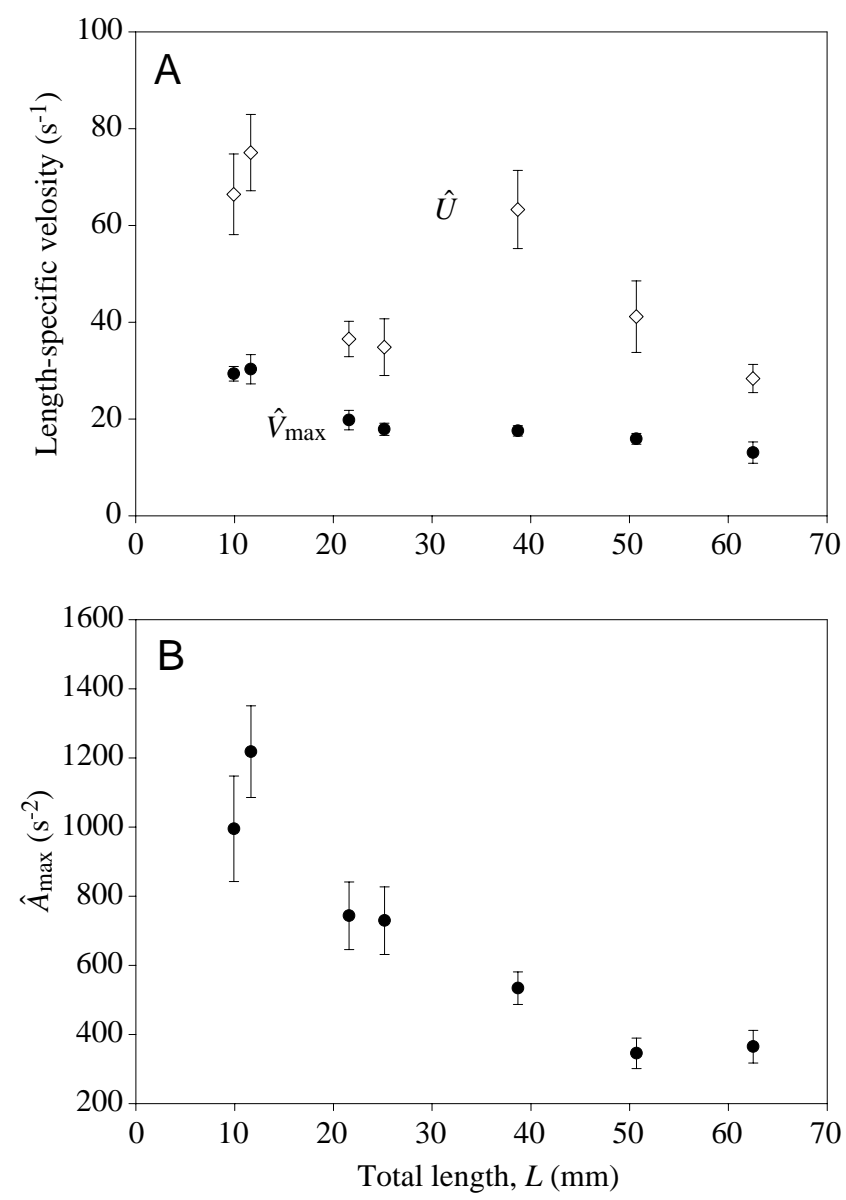

Fig. 9. (A) Length-specific maximum velocity $\hat{V}_{\max }$ (filled circles) and velocity of wave of spine curvature $\hat{U}$ (open diamonds) as a function of body length. (B) Length-specific maximum acceleration $\hat{A}_{\text {max }}$. Values were calculated for the first tail-beat of each fast-start and represent the mean ( \pm S.E.M.) from the five most powerful faststarts in each size group.

to occur in the front region of the trunk muscle $0.3<\hat{\imath}<0.5$. Posterior to this region, there was a gradual decrease in strain to a low value of $\varepsilon \approx 0.03$ at $0.8 L$.

Table 2. Integrated mass-specific muscle power output for the whole-fish white muscle, hydrodynamic power requirements and hydrodynamic efficiency during the first tail-beat of the fast-start

\begin{tabular}{|c|c|c|c|c|}
\hline \multirow{2}{*}{$\begin{array}{l}\text { Total length, } L \\
\text { (m) }\end{array}$} & \multicolumn{2}{|c|}{ Muscle $\overline{P_{\mathrm{m}, \mathrm{t}}}$} & \multirow{2}{*}{$\begin{array}{l}\text { Hydrodynamic } \overline{P_{\mathrm{i}}} \\
\quad\left(\mathrm{W} \mathrm{kg}^{-1} \text { fish }\right)\end{array}$} & \multirow[b]{2}{*}{ Efficiency, $\eta$} \\
\hline & (W kg-1 muscle) & (W kg ${ }^{-1}$ fish) & & \\
\hline 0.010 & $6.51 \pm 2.09$ & $1.28 \pm 0.41$ & $1.26 \pm 0.16$ & $0.83 \pm 0.06$ \\
\hline 0.022 & $29.72 \pm 5.84$ & $6.03 \pm 1.19$ & $2.83 \pm 0.60$ & $0.26 \pm 0.02$ \\
\hline 0.025 & $29.55 \pm 7.69$ & $6.29 \pm 1.64$ & $3.12 \pm 0.79$ & $0.48 \pm 0.20$ \\
\hline 0.039 & $43.86 \pm 13.82$ & $12.59 \pm 3.97$ & $5.38 \pm 0.50$ & $0.44 \pm 0.13$ \\
\hline 0.063 & $55.99 \pm 8.31$ & $16.57 \pm 2.46$ & $6.97 \pm 1.13$ & $0.36 \pm 0.10$ \\
\hline
\end{tabular}

Values are given as mean \pm S.E.M. $(N=5)$.

$\overline{P_{\mathrm{m}, \mathrm{t}}}$, mean mass-specific muscle power output; $\overline{P_{\mathrm{i}}}$, mean power requirements. 
Fast-start biomechanics during ontogeny in carp 3063

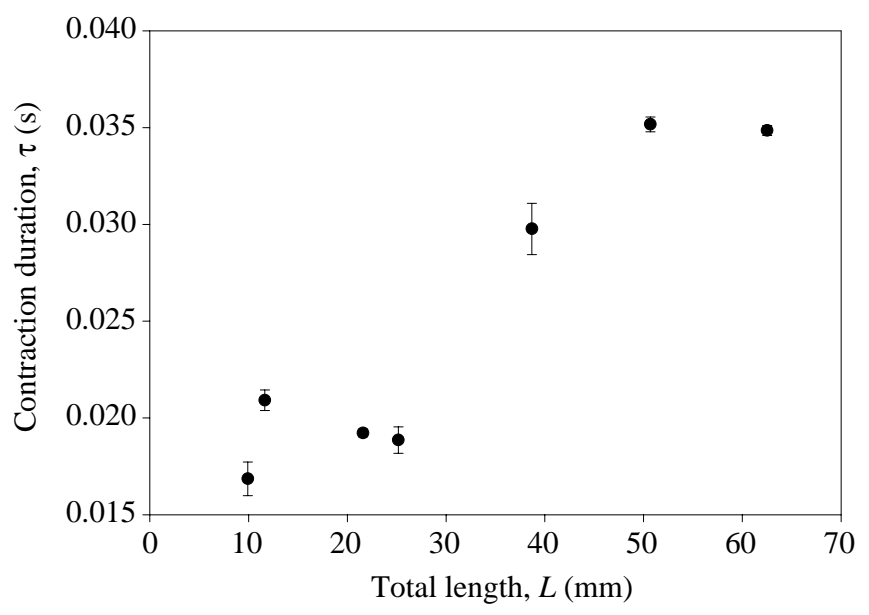

Fig. 10. Duration of the initial muscle contraction for each fast-start. Symbols denote the mean ( \pm S.E.M.) from the five most powerful fast-starts in each size group.

\section{Fast-start muscle mechanics}

The mean muscle stress $\bar{\sigma}$ and mass-specific power output $\bar{P}$, integrated over the first tail-beat, were calculated for each segment of the body trunk for each fast-start sequence (Fig. 13; Table 2). $\bar{P}$ was greatest in the mid region of the fish $0.3<\hat{l}<0.5$ (for $L \geqslant 12 \mathrm{~mm}$ ) and decreased towards the caudal fibres at $0.8 L$. There was an increase in the power output with increasing body length. In contrast, $\bar{\sigma}$ was smallest in the mid region of the fish $0.3<\hat{l}<0.5$ and increased towards the caudal fibres at $0.8 L$. There was an increase in the stress with increasing body length. Analysis of covariance showed that both body length and longitudinal position had a significant

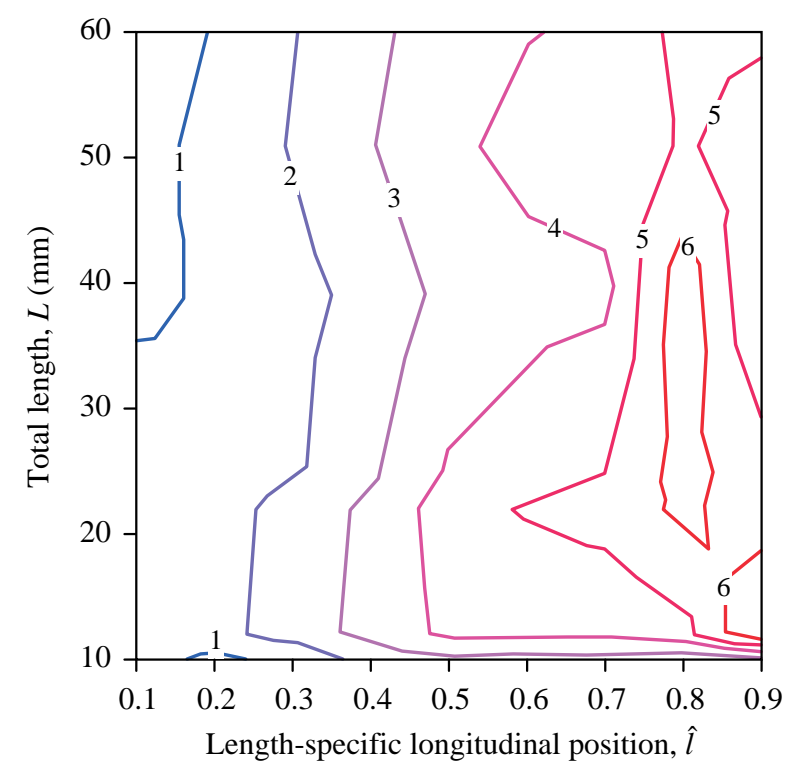

Fig. 11. Spine curvatures during the initial tail-beat of the fast-start. The contour height (1-6) shows the maximum change in lengthspecific spine curvature $\hat{c}$ as a function of both length-specific longitudinal position $\hat{l}$ and total length $L$.
Table 3. Results of the analysis of covariance for mean muscle stress and muscle-mass-specific power output during first tailbeat of a fast-start

\begin{tabular}{llrrc}
\hline Variable & \multicolumn{1}{c}{ Source } & d.f. & $F$-ratio & $P$ \\
\hline $\bar{\sigma}\left(\mathrm{kN} \mathrm{m}^{-2}\right)$ & Body length & 1 & 170.81 & $<0.001$ \\
& Longitudinal position & 1 & 20.16 & $<0.001$ \\
& Error & 240 & & \\
$\bar{P}\left(\mathrm{~W} \mathrm{~kg}^{-1}\right)$ & Body length & 1 & 81.79 & $<0.001$ \\
& Longitudinal position & 1 & 26.14 & $<0.001$ \\
& Error & 240 & &
\end{tabular}

$\bar{\sigma}$, mean muscle stress; $\bar{P}$, mean muscle-mass-specific power output.

effect on the mean stress $(P<0.001)$ and the mean mass-specific power output $(P<0.001)$ (Table 3$)$.

\section{Discussion}

Size, speed and Reynolds number regime

During ontogeny, as the body length increases in the carp, so does maximum fast-start velocity. Therefore, the Reynolds number regime in which the fish swim must also have increased. Using a kinematic viscosity of $v=1.004 \times 10^{6} \mathrm{~m}^{2} \mathrm{~s}^{-1}$ appropriate to fresh water at $20^{\circ} \mathrm{C}$ and equation 1 , the maximum Reynolds number reached during the fast-starts increased from 2900 for the $10 \mathrm{~mm}$ fish to 49200 for the $63 \mathrm{~mm}$ fish. All the fast-starts were for fish starting from rest, with an initial $R e=0$; however, all these fish would quickly have entered the regime $(R e>300$; Fuiman and Batty, 1997) where inertial forces dominated the hydrodynamics.

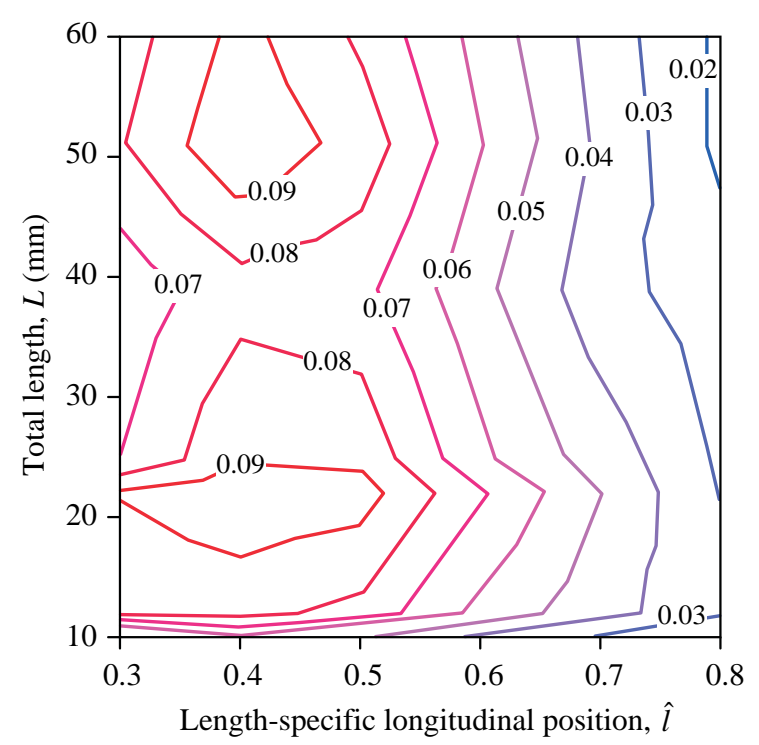

Fig. 12. White muscle strain during the initial tail-beat of the faststart. The contour height (0.02-0.09) shows the maximum change in white muscle strain as a function of both length-specific longitudinal position $\hat{l}$ and total length $L$. 

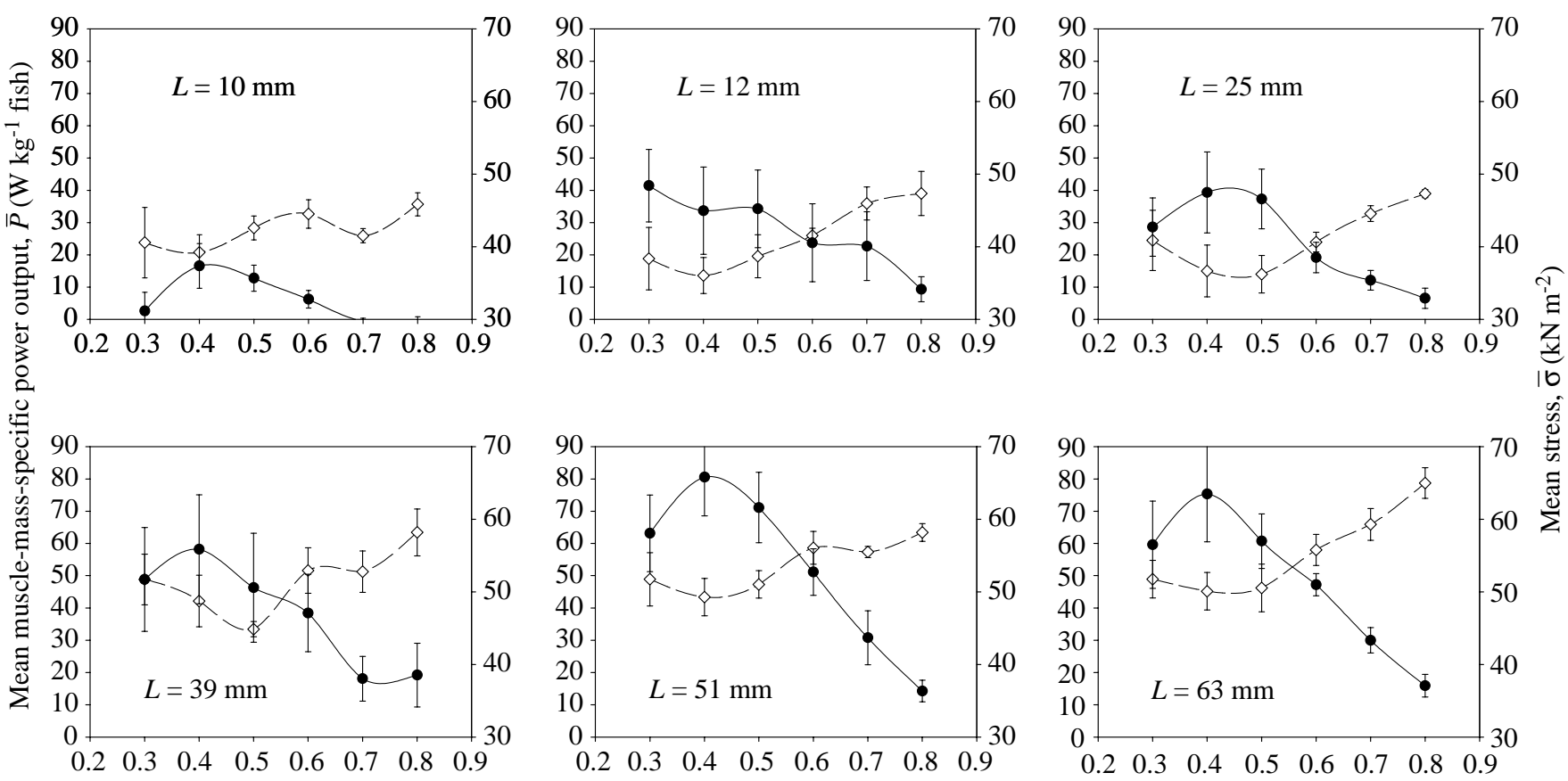

Length-specific longitudinal position, $\hat{l}$

Fig. 13. Mean muscle-mass-specific power output $\bar{P}\left(\mathrm{~W} \mathrm{~kg}^{-1}\right)$ (filled circles, solid line, left axes) and mean muscle stress $\bar{\sigma}\left(\mathrm{kN} \mathrm{m}^{-2}\right)($ open diamonds, dashed line, right axes) integrated over the first muscle contraction cycle during each fast-start as a function of length-specific longitudinal position $\hat{l}$. A separate plot is shown for each size group. Symbols denote the mean $( \pm$ S.E.M.) from the five fast-starts with the greatest hydrodynamic power requirements in each size group. Lines are spline fits to clarify the trends. $L$, total body length.

The kinematics of body bending for all the size classes of these fish, apart from the $10 \mathrm{~mm}$ group, were similar (Fig. 11), suggesting that the swimming style is maintained across this developmental range. The spine curvatures for the $10 \mathrm{~mm}$ fish were similar to those of the larger fish for $\hat{l}<0.4$, but their curvatures increased little beyond this point towards the tail (Fig. 11). All the larger sizes, in contrast, showed increasing spine curvatures as far as the $\hat{l}<0.8$ position. The kinematics of these fast-starts were similar to those for steady swimming in that waves of bending travelled along the body and the amplitude of changes in spine curvature increased in a posterior direction along the fish. The difference in kinematics between the $10 \mathrm{~mm}$ fish and the larger classes may reflect an increasing rigidity of the anterior part of the body (Osse, 1990).

\section{Muscle function along the body}

A characteristic feature of the muscle dynamics of fast-starts is that there is a drop in mean mass-specific power output between the longitudinal positions $0.4 L$ and $0.8 L$, and there is a corresponding increase in muscle stress between these locations (Fig. 13). For instance, for the $63 \mathrm{~mm}$ fish, the $0.8 \mathrm{~L}$ values for mass-specific power output are $21 \%$ and for stress are $130 \%$ of their respective values at $0.4 \mathrm{~L}$.

During fast-starts, the majority of the hydrodynamic power is delivered into the water from the caudal region of the fish (Weihs, 1973; Frith and Blake, 1991), whereas the majority of the white myotomal muscle available for power generation is situated in the central region $0.3<\hat{l}<0.5$ of the body. Muscle in the caudal region of the fish must act to increase the body stiffness that is needed to transmit power from the main muscle bulk to the tail. Caudal muscle fibres must therefore act as stiff elements, and this is achieved by producing higher forces at the expense of generating a high power output. Whole-body 'work loop' experiments have shown that increases in body stiffness do result from increases in muscle stiffness and decreases in the work done by the muscles (Long and Nipper, 1996).

Changes in muscle power and force production along the fish during fast-starts occur as a result of the decreasing strain and, therefore, strain rate experienced by the muscle (Fig. 12; Wakeling and Johnston, 1998, 1999a). Longitudinal changes in force and power production have been predicted during steady swimming as a result of phase changes between the activation and shortening of the muscle fibres (for a review, see Wardle et al., 1995). However, during fast-starts, the muscle starts to shorten immediately after activation at all longitudinal sites (Wakeling and Johnston, 1999c). The phase changes found in steady swimming between activation and shortening are not required to explain the difference in muscle function during fast-starts.

The time-dependent contractile properties of the white myotomal muscle have been shown to be slower in caudal than in rostral myotomes for cod Gadus morhua (Davies et 
al., 1995) and saithe Pollachius virens (Altringham et al., 1993), although no significant differences were found between these sites in the sculpin Myoxocephalus scorpius (Johnston et al., 1995; James and Johnston, 1998a). Slower contractile properties of the caudal myotomes predispose them to slow strain rates and therefore higher force production. However, the reduction in mass-specific power output resulting from the different contractile properties of the muscle between rostral and caudal sites (to $49 \%$ in the caudal myotomes for the saithe; Altringham et al., 1993) is less than half of that which can be explained by the body kinematics and longitudinal change in muscle strain rate during fast-starts and may not even be significant (James and Johnston, 1999a). In contrast, the longitudinal change in muscle strain along the fish plays a major role in balancing muscle function between force and power production during fast-starts.

\section{Scaling effects on fast-start kinematics}

The fish were characteristically stationary with their body straight prior to the initiation of a fast-start. An initial rapid bending of the body marked the beginning of each fast-start. This body bending is the result of the interaction between torque being generated by the myotomal muscle and the resistance to bending, which is predominantly the inertia of the body and the added mass of water (Wakeling and Johnston, $1999 \mathrm{c})$. The rate of bending can be expressed in terms of scaling relationships for geometric fish.

The angular acceleration $\alpha$ for bending the spine about a vertical axis running through the spine is:

$$
\alpha \propto T / I,
$$

where $T$ is the muscle torque and $I$ is the net moment of inertia of the front and back regions of the fish about that axis. The muscle torque is the product of the muscle force (proportional to its cross-sectional area) and the distance of that force from the spine (related to a length term). Expressing torque in scaling terms gives $T \propto L^{2} L \propto L^{3}$. The moment of inertia is the product of mass (proportional to its volume) and the distance of that mass from the axis of rotation (related to a length term). Therefore, the scaling representation of inertia can be expressed as $I \propto L^{3} L \propto L^{4}$.

Size-related changes in the angular rate of bending can be estimated from the scaling relationship:

$$
\alpha \propto L^{3} / L^{4} \propto 1 / L .
$$

Therefore, the angular acceleration of spine bending should be inversely proportional to the fish length, i.e. large fish should bend more slowly.

An equation for angular motion states that:

$$
\omega_{2}^{2}=\omega_{1}^{2}+2 \alpha \theta,
$$

where the angular quantities are initial velocity $\omega_{1}$, final velocity $\omega_{2}$, acceleration $\alpha$ and displacement $\theta$. The white muscle strain rate $\dot{\varepsilon}_{\mathrm{w}}$ is initially zero and, during the first tailbeat, is proportional to the rate of change of spine curvature (see equation 3). Equation 7 shows that $\dot{\varepsilon}_{\mathrm{w}}$ is related to the angular acceleration by:

$$
\dot{\varepsilon}_{\mathrm{w}} \propto \alpha \theta,
$$

where $\theta$ is the angle through which the spine has bent. During these fast-starts, the muscle strain is relatively constant through the range of fish lengths for any longitudinal position along the body. Therefore, assuming that $\theta$ is constant and combining equations 6 and 8 , the scaling relationship for muscle strain rate becomes:

$$
\dot{\varepsilon}_{\mathrm{W}} \propto 1 / \sqrt{L} .
$$

The duration of fast-starts in adult rainbow trout Oncorhynchus mykiss scales as $L^{2.36}$ and $L^{1.73}$ for ' $\mathrm{C}$ ' and ' $\mathrm{S}$ ' starts, respectively (Webb, 1976). Data were not given for the muscle strains for these fast-starts, but if the strains were independent of length, then these scaling relationships satisfy the above predictions. The muscle shortening durations for adult sculpin Myoxocephalus scorpius (James and Johnston, 1998b) also satisfy the scaling predictions for fast-starts; muscle strain estimates were given for these fish; however, without data on the chordwise location of the muscle strain, estimates of the change in mean muscle strain with body length (Wakeling and Johnston, 1999a) cannot be made. Large changes in body morphology during early larval development result in allometric scaling, and early larval fast-starts may not, therefore, follow these patterns (equation 9). Indeed, $15 \mathrm{~mm}$ chinook salmon Oncorhynchus tshawytscha have a yolk sac comprising $75 \%$ of their mass which has been absorbed by the time the fish achieves a length of $35 \mathrm{~mm}$; during development over this size range, there was a decrease in the duration of body bending (Hale, 1996).

Data from the present study show that there is an increase in contraction duration with increasing body length (Fig. 10) which corresponds to a decrease in mean contraction velocity of the white muscle fibres, given that the muscle strains are similar at different sizes (Fig. 12). The scaling relationship (equation 9) is based on a number of assumptions, including geometric similarity of the fish in terms of both shape and muscle distribution, and also assuming identical muscle contractile properties and activation patterns. These assumptions are not valid across the sizes of carp in the present study, but the main conclusions of the scaling predictions hold true. Increases in body length constrain the fish to bend less rapidly because the cross-sectional muscle area, and hence force production, does not increase at the same rate as the inertial mass that resists the bending.

\section{Muscle performance during ontogeny}

As fish length increases, the changing interaction between the muscle bulk and the body results in a decrease in strain rate during the initial tail-beat of the fast-start. For the case of the carp in the present study, this change occurs as an increase in the contraction duration coupled to an invariant peak muscle strain. During the brief contractions used for fast-starts, the decreasing strain rate that occurs with increasing body length causes the following changes in muscle contractile dynamics: 


\section{J. M. WaKeling, K. M. KeMP AND I. A. Johnston}

the activation rate increases, the maximum muscle force increases, the time course for force production increases and the rate of deactivation decreases in relation to the rate of activation (Wakeling and Johnston, 1999b). The muscle power output is the product of its force and shortening velocity, and the mass-specific power showed an increase with increasing body length across the size range of carp from this study.

The increases in muscle stress and mass-specific power output with body length shown in Fig. 13 are due entirely to the changes in contraction regime because the intrinsic muscle contractile properties were assumed to be invariant during ontogeny for these fish. The change in muscle contraction regime is sufficient to account for a $42 \%$ increase in muscle stress and a $386 \%$ increase in mass-specific power output. However, changes in muscle composition during ontogeny may additionally predispose the muscle to an increased performance for larger fish. Indeed, the myosin ATPase for carp myotomal muscle tends to decrease in activity with ontogeny across a similar size range to that used in this study. However, this decrease was not significant (N. J. Cole and I. A. Johnston, in preparation). In other species of fish, the length-dependent changes in twitch contraction times (Archer et al., 1990; Videler and Wardle, 1991) and maximum unloaded shortening velocity (James and Johnston, 1998b) may only explain an $11 \%$ change in contractile variables for the $50 \mathrm{~mm}$ range of fish lengths appropriate to the present study. The changes in muscle stress and mass-specific power output due to the different length-dependent contraction regimes therefore make a much larger contribution to the developmental changes in muscle performance than do the changes in muscle composition that occur during ontogeny.

\section{Total muscle power output and hydrodynamic efficiency}

During swimming, energy is expended for both normal and tangential components of the motion. However, it is only the tangential component of the propulsive force that causes acceleration of the fish in its direction of travel. The hydrodynamic efficiency $\eta$ is the ratio of the tangential hydrodynamic force to the total hydrodynamic force. During fast-starts in pike Esox lucius, $\eta$ was estimated to be between 0.16 and 0.39 and took a mean value of $0.32 \pm 0.04$ (mean \pm S.E.M., $N=6$ ) (Frith and Blake, 1995). The tangential inertial power $\overline{P_{\mathrm{i}}}$ requirements can be used as an estimate of the 'useful' tangential hydrodynamic power, and $\eta$ for these pike was $0.31 \pm 0.05$ using this second estimate (data in Frith and Blake, 1995).

The total hydrodynamic power requirements are generated by the mechanical power output of the muscle. Indeed, the maximum fast-start performance is limited by the maximum muscle power available (Wakeling and Johnston, 1998). The total mechanical power output from the muscles $\overline{P_{\mathrm{m}, \mathrm{t}}}$ (Table 2 ) can therefore be used as an estimate of the total hydrodynamic power requirements for the fast-start. Thus:

$$
\eta=\overline{P_{\mathrm{i}}} / \overline{P_{\mathrm{m}, \mathrm{t}}} .
$$

The mean hydrodynamic efficiency for the fast-starts in this study $(L \geqslant 12 \mathrm{~mm})$ was $0.39 \pm 0.05 \quad(N=24)$ and was not significantly different from the value for fast-starting pike derived purely from hydrodynamics (Frith and Blake, 1995). There was no significant relationship between hydrodynamic efficiency and body length across this range of body length, and this concurs with the body bending kinematics also being maintained across this range (Fig. 11).

This work was supported by a non-thematic grant (GR3/11028) from the Natural Environment Research Council of the UK.

\section{References}

Altringham, J. D., Wardle, C. S. and Smith, C. I. (1993). Myotomal muscle function at different locations in the body of a swimming fish. J. Exp. Biol. 182, 191-206.

Archer, S. D., Altringham, J. D. and Johnston, I. A. (1990). Scaling effects on the neuromuscular system, twitch kinetics and morphometrics of the cod Gadus morhua. Mar. Behav. Physiol. 17, 137-146.

Beamish, F. W. H. (1978). Swimming capacity. In Fish Physiology, vol. 7, Locomotion (ed. W. S. Hoar and D. J. Randall), pp. 101-187. London: Academic Press.

Curtin, N. A. and Woledge, R. C. (1988). Power output and force-velocity relationship of live fibres from white myotomal muscle of the dogfish, Scyliorhinus canicula. J. Exp. Biol. 140, 187-197.

Davies, M. L. F., Johnston, I. A. and van de Wal, J. (1995). Muscle fibres in rostral and caudal myotomes of the Atlantic cod (Gadus morhua L.) have different mechanical properties. Physiol. Zool. 68 , 673-697.

Frith, H. R. and Blake, R. W. (1991). Mechanics of the startle response in the northern pike, Esox lucius. Can. J. Zool. 69, 2831-2839.

Frith, H. R. and Blake, R. W. (1995). The mechanical power output and hydromechanical efficiency of northern pike (Esox lucius) faststarts. J. Exp. Biol. 198, 1863-1873.

Fuiman, L. A. and Batty, R. S. (1997). What a drag it is getting cold: partitioning the physical and physiological effects of temperature on fish swimming. J. Exp. Biol. 200, 1745-1755.

Hale, M. E. (1996). The development of fast-start performance in fishes: escape kinematics of the chinook salmon (Oncorhynchus tshawytscha). Am. Zool. 36, 695-709.

James, R. S. and Johnston, I. A. (1998a). Scaling of intrinsic contractile properties and myofibrillar protein composition of fastmuscle in the fish Myxocephalus scorpius L. J. Exp. Biol. 201, 901-912.

James, R. S. and Johnston, I. A. (1998b). Scaling of muscle performance during escape responses in the fish Myoxocephalus scorpius L. J. Exp. Biol. 201, 913-923.

Johnston, I. A., van Leeuwen, J. L., Davies, M. L. and Beddow, T. (1995). How fish power predation fast-starts. J. Exp. Biol. 198, 1851-1861.

Lighthill, M. J. (1969). Hydromechanics of aquatic animal propulsion. Annu. Rev. Fluid Mech. 1, 413-446.

Lighthill, M. J. (1970). Aquatic animal propulsion of high hydromechanical efficiency. J. Fluid Mech. 44, 265-301.

Long, J. R. and Nipper, K. S. (1996). The importance of body stiffness in undulatory propulsion. Am. Zool. 36, 678-694. 
Magnuson, J. J. (1978). Locomotion by scombriod fishes: hydrodynamics, morphology and behaviour. In Fish Physiology, vol. 7 (ed. W. S. Hoar and D. J. Randall), pp. 239-313. London: Academic Press.

Mendez, J. and Keys, A. (1960). Density and composition of mammalian muscle. Metabolism 9, 184-188.

Osse, J. W. M. (1990). Form changes in fish larvae in relation to changing demands on function. Neth. J. Zool. 40, 362-385.

Temple, G. K. and Johnston, I. A. (1998). Testing hypotheses concerning the phenotypic plasticity of escape performance in fish of the family Cottidae. J. Exp. Biol. 201, 317-331.

Videler, J. J. (1993). Fish Swimming. London: Chapman \& Hall.

Videler, J. J. and Wardle, C. S. (1991). Fish swimming stride by stride: speed limits and endurance. Rev. Fish Biol. Fish. 1, 23-40.

Wakeling, J. M. and Johnston, I. A. (1998). Muscle power output limits fast-start performance in fish. J. Exp. Biol. 201, 1505-1526.

Wakeling, J. M. and Johnston, I. A. (1999a). White muscle strain in the common carp and red to white muscle gearing ratios in fish. J. Exp. Biol. 202, 521-528.

Wakeling, J. M. and Johnston, I. A. (1999b). Predicting muscle force generation during fast-starts for the common carp Cyprinus carpio. J. Comp. Physiol. B 169, 391-401.
Wakeling, J. M. and Johnston, I. A. (1999c). A hydrodynamic resistance model of bending can explain key features of fish faststarts. J. Exp. Biol. 202, 675-682.

Wardle, C. S., Videler, J. J. and Altringham, J. D. (1995). Review: Tuning in to fish swimming waves: body form, swimming mode and muscle function. J. Exp. Biol. 198, 1629-1636.

Webb, P. W. (1976). The effect of size on the fast-start performance of rainbow trout Salmo gairdneri and a consideration of piscivorous predator-prey interactions. J. Exp. Biol. 65, 157-177.

Webb, P. W. (1978). Fast-start performance and body form in seven species of teleost fish. J. Exp. Biol. 74, 211-226.

Webb, P. W. and Weihs, D. (1986). Functional locomotor morphology of early life history stages of fishes. Trans. Am. Fish. Soc. 115, 115-127.

Weihs, D. (1973). The mechanism of rapid starting of slender fish. Biorheology 10, 343-350.

Williams, P. J. and Brown, J. A. (1992). Developmental changes in the escape response of larval winter flounder Pleuronectes americanus from hatch through metamorphosis. Mar. Ecol. Prog. Ser. 88, 185-193.

Wu, T. Y. (1977). Introduction to the scaling of aquatic animal locomotion. In Scale Effects in Animal Locomotion (ed. T. J. Pedley), pp. 203-232. New York: Academic Press. 